\title{
Nanoplasmonic discrimination of organic solvents using a bimetallic optical tongue
}

Justin R. Sperling, Gerard Macias, Glenn A. Burley, Steven L. Neale, Alasdair W. Clark

Justin R. Sperling, Gerard Macias, Glenn A. Burley, Steven L. Neale, Alasdair W. Clark, "Nanoplasmonic discrimination of organic solvents using a bimetallic optical tongue," Proc. SPIE 10895, Frontiers in Biological Detection: From Nanosensors to Systems XI, 108950G (7 March 2019); doi: $10.1117 / 12.2507275$

SPIE. Event: SPIE BiOS, 2019, San Francisco, California, United States 


\title{
Nanoplasmonic Discrimination of Organic Solvents Using a Bimetallic Optical Tongue
}

\author{
Justin R. Sperling, ${ }^{\mathrm{a}}$ Gerard Macias, ${ }^{\mathrm{a}}$ Glenn A. Burley, ${ }^{\mathrm{b}}$ \\ Steven L. Neale, ${ }^{\mathrm{a}}$ and Alasdair W. Clark ${ }^{\mathrm{a}}$ \\ ${ }^{a}$ School of Engineering, University of Glasgow, Rankine Building, Oakfield Avenue, Glasgow, UK. \\ E-mail: Alasdair.clark@glasgow.ac.uk \\ ${ }^{\mathrm{b}}$ WestCHEM \& Department of Pure \& Applied Chemistry, University of Strathclyde, \\ 295 Cathedral Street, Glasgow, G1 1XL, UK. E-mail: glenn.burley@strath.ac.uk
}

\section{ABSTRACT}

Optical sensor arrays serve as excellent tools for the recognition and discrimination of a variety of liquid and gas mixtures. They achieve this via pattern-based recognition from signals across multiple sensing regions, where each region is modified to produce a different interaction, such as partial-selectivity, with desired analytes. As their use progresses towards rapid, highly personalized diagnosis and component identification devices, reduction in complexity and data-acquisition time is key. One way to achieve this is through reducing the number of elements in the array without compromising the differential capabilities of the device.

Here, we present a device with elements consisting of plasmonic sensors of two superimposed plasmonic nanoarrays; one fabricated using gold and the other aluminum. Each material produces a distinct plasmonic response while also allowing us to selectively functionalize each pattern with a different 'sensing chemistry.' This allows for the development of different partially-selective elements, via modification with functional thiols and silanes, respectively.

Since optical sensing arrays of this type require multiple sensing regions, each producing a different optical response, our bimetallic method results in twice as much data from one measurement, providing the same amount of data necessary to allow for successful differentiation with fewer elements in the sensing array. We demonstrate that by altering the surface chemistry of the nanostructures we can tune their partial selectivity to organic solvents. We believe this technology could be useful in areas that rely on assays for simultaneous determination of multiple analytes, such as the medical, food \& drug, and security industries.

Keywords: plasmonic, optoelectronic, LSPR, bimetallic, optical tongue

\section{INTRODUCTION}

Chromatography — the standard technology used to accurately identify, detect, and quantify components in a gas or liquid mixture - requires efficient separation of the components prior to analysis. ${ }^{1-2}$ The equipment necessary to achieve adequate separation is bulky, complex, and costly; and the processes used are time-consuming and highly wasteful. ${ }^{3}$ Together, these problems with chromatography make it unsuitable for portable applications such as point-of-care diagnostics and the food and drug industry. Recent efforts have turned to designing detection and identification technologies modelled on the animals senses of smell and taste $e^{4-7}$-accurately referred to as optical ${ }^{8}$ and electronic ${ }^{7,}{ }^{9}$ tongues/noses. Modelling technology in this way has allowed for the development of portable systems capable of the accurate identification of mixtures that can be fabricated relatively cheaply in bulk and do not require component isolation, ${ }^{10}$ making them more widely applicable $4,7,11,6,12-13$.

As a mimicry of our perception of taste and smell, optical and electronic tongues/noses work similarly to how our senses do; rather than relying on separation of a mixture's components, identification is a product of a combined response between multiple partially selective 'sensors' that result in a distinct pattern. ${ }^{14-15}$ This mechanism requires multiple cross-reacting 
sensors trained to detect patterns rather than specific analytes. ${ }^{15}$ As more sensing regions are added to the system, these artificial tongue/nose devices becomes more accurate at differentiating between different tastes/smells (given that the ratio of samples to variables is above six). ${ }^{16}$

One of the most common methods to provide partial-selectivity in electronic noses is the use of metal oxides doped with platinum noble metal catalysts; ${ }^{11}$ however, these catalysts are expensive and thus limit the scope for practical, commercially-viable devices. Instead, devices based on plasmonic gold $\mathrm{Au}$ ) nanostructures have been recently used in electronic nose applications. ${ }^{17}$ Subject to the phenomena known as localised surface plasmon resonance, the optical response of an array of Au nanostructures is highly susceptible to changes in the local environment. ${ }^{18}$ Devices utilizing Au nanostructures take advantage of this by having multiple sensing regions of $\mathrm{Au}$ nanostructures, each uniquely functionalized with a modified surface ligand, yielding multiple partially-selective responses to a particular mixture. ${ }^{19}$ Each modified Au region of the sensor array provides one resonance peak as part of the partially selective process. Thus, current electronic nose and tongue devices typically operate with up to 32 sensing regions and weight about $1 \mathrm{~kg}$ (Cyranose 320 Device), which limits their widespread use. ${ }^{19-22}$

Here, we present a novel device capable of obtaining two partially selective data points from one measurement. Our device consists of two superimposed nanoplasmonic arrays featuring distinct metals: Au and aluminum (Al). In addition to their different plasmonic responses, both metals are susceptible to different surface chemistries modifications, which allows for the selective functionalization of both arrays independent of one another (Au by thiols ${ }^{23-24}$ and $\mathrm{Al}$ by silanes ${ }^{24-25}$ ). Our results show that functionalization can be used to tune the partial selectivity of the nanostructures to acetone, methanol $(\mathrm{MeOH})$, ethanol $(\mathrm{EtOH})$, isopropyl alcohol (IPA) in varying concentrations (volume per volume (v/v)) in water. The two plasmonic peaks obtained from one measurement, combined with the ability to modify the partial selectivity of the sensing regions by utilizing different thiols and silanes, allows us to condense two sensing regions of a standard plasmonic tongue/nose device into one region. This effectively reduces the number of partially selective elements in half, reducing the size of the device and number of elements to probe (and thus time to acquire data) by half without compromising the identification and classification capabilities of the device.

\section{EXPERIMENTAL SECTION}

\subsection{Device Fabrication}

Devices were fabricated using electron-beam lithography and metal-evaporation. A resist bi-layer of $4 \%$ poly(methyl methacrylate) (PMMA) 2010 resist and 2.5\% PMMA 2041 resist (total thickness $150 \mathrm{~nm}$ ) was patterned using a Vistec VB6 Ultra High Resolution Extra Wide Field electron beam lithography tool. Following development of the pattern, a $2 / 50 \mathrm{~nm} \mathrm{Ti} / \mathrm{Au}$ layer was evaporated onto the sample using a Plassys MEB 400S/550S electron-beam evaporation tool. These fabrication steps were then repeated to align $50 \mathrm{~nm}$ thick Al nanostructures to the existing Au structures.

\subsection{Surface Functionalization}

Silane and thiol chemistries were used to modify the $\mathrm{Al}$ and $\mathrm{Au}$ nanostructures, respectively. ${ }^{23-25,}{ }^{28}$ Three pairs of chemistries were used. For the first pair of chemistries (Figure 1a), Al and Au nanostructures were unmodified and thus retained their native chemistries. For the second pair of chemistries (Figure 1b), Au was modified with 1-decanethiol (DT, Sigma-Aldrich) and Al was modified with hexamethyldisilazane (HMDS, Sigma-Aldrich). Exposed sensor regions were first placed in an ethanolic solution containing $10 \mathrm{mM}$ DT for 24 hours, rinsed with fresh ethanol, and nitrogen dried. HMDS was then spun on to these same exposed regions at 4000 revolutions per minute for 60 seconds and allowed to airdry for 2 minutes. For the third set of chemistries (Figure 1c), exposed sensor regions were immersed in a $10 \mathrm{mM}$ ethanolic solution of 1H,1H,2H,2H-perfluoro-1-decanethiol (PFDT, Sigma Aldrich) for 24 hours, rinsed three times with ethanol, and nitrogen air-dried. The exposed regions were then immersed in $0.5 \%$ solution (by volume) of 2-[methoxy (polyethyleneoxy)6-9 propyl] trimethoxysilane (PEG69, Sigma-Aldrich) in toluene for 1 hour, rinsed three times with toluene, rinsed three times with deionized water, nitrogen air-dried, and oven-baked at $100^{\circ} \mathrm{C}$ for 30 minutes. 

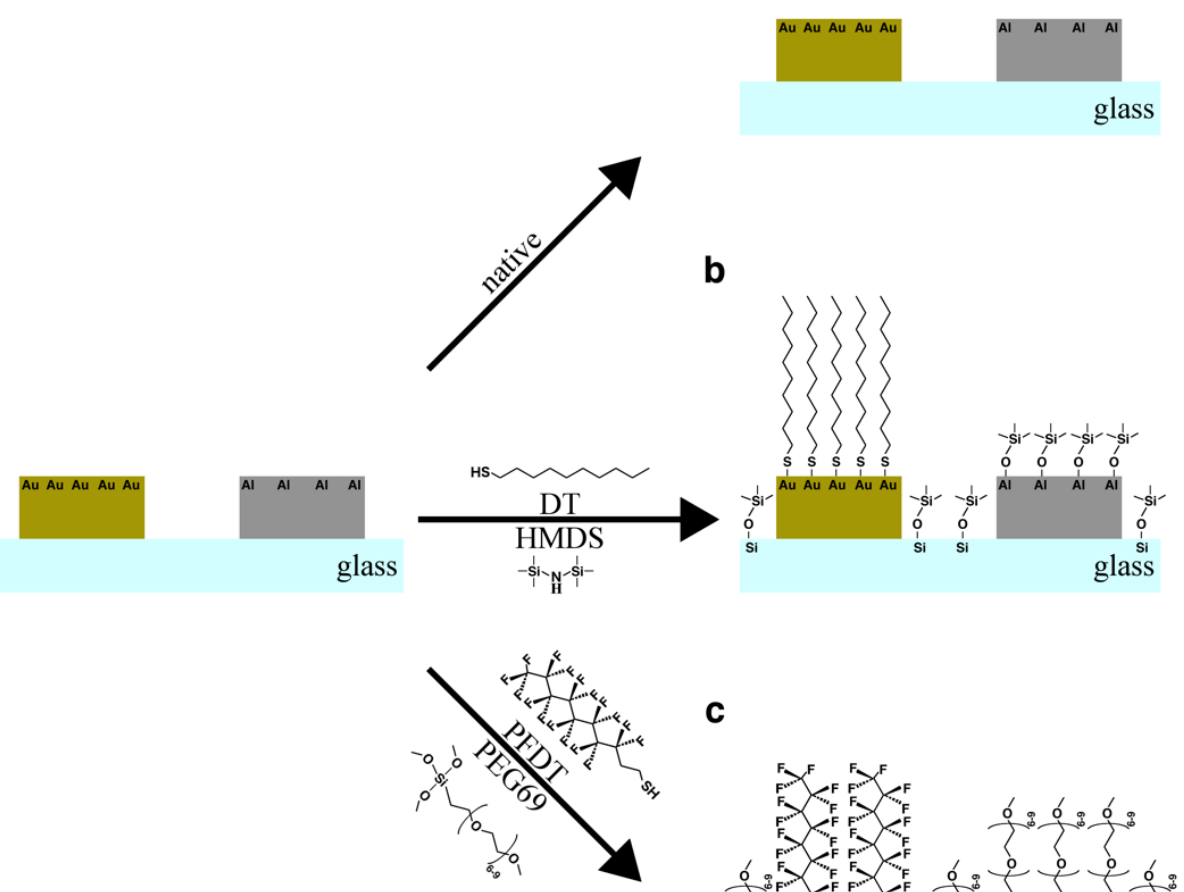

C

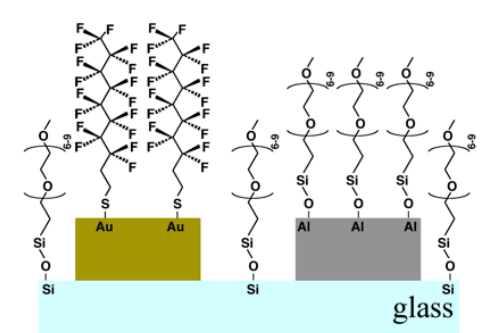

Figure 1: Surface chemistry modifications used in the optical tongue: (a) native Al / Au, (b) Al-HMDS / Au-DT, and (c) Al-PEG69 / Au-PFDT.

\subsection{Solution Preparation}

Solutions of $5 \%, 10 \%, 25 \%$, and $50 \%$ (by volume) of acetone, methanol (MeOH), ethanol (EtOH), and isopropyl alcohol (IPA) in water were prepared.

\subsection{Experimental Setup}

Samples were placed in a chamber (polydimethylsiloxane (PDMS) on a glass slide) filled with each solution. Real-time transmission measurements ( $0.5 \mathrm{~nm}$ resolution) were taken using a custom-built microspectrophotometer (Figure 2). Light from a VIS-NIR light source (tungsten-halogen 400 to $1200 \mathrm{~nm}$ wavelength) was used to probe the sensor. A 10x objective was used to couple the transmitted light into an optical-fiber attached to a StellarNet Microspectrophotometer (StellarNet Blue Wave). 


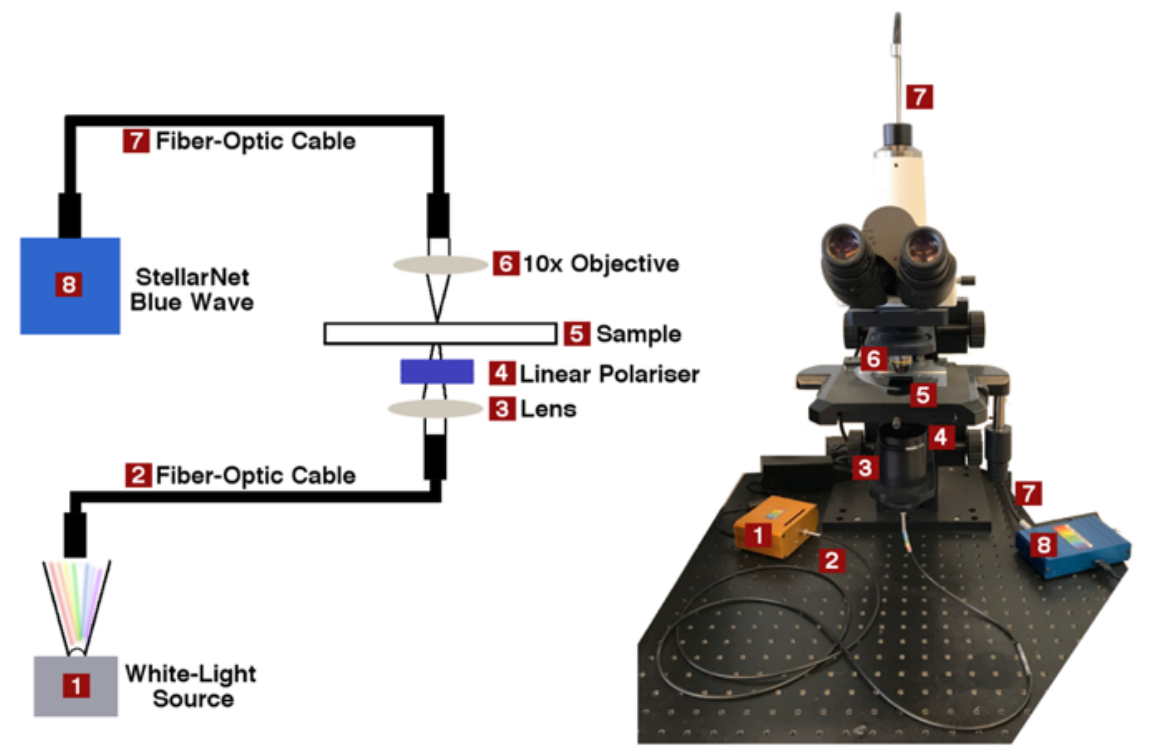

Figure 2: Custom-built microspectrophotometer setup.

\subsection{Data Analysis}

MATLAB was used to analyze the transmission spectra. The transmission spectrum was smoothed (20 points, meanaverage smoothing) and interpolated (from $0.5 \mathrm{~nm}$ to $0.01 \mathrm{~nm}$ ). The peak position value of the two minima peaks was determined. Principal component analysis (PCA) was then used to analyze the resulting transmission peaks. ${ }^{29}$ The inherent PCA function in MATLAB was then used to calculate and plot PCA results. The wavelength of the resonant peaks for each chemistry (Au, Al, Au-DT, Al-HMDS, Au-PFDT, Al-PEG6-9) were treated as separate columns in the PCA.

\section{RESULTS AND DISCUSSION}

\subsection{Design}

The optical tongue design consists of three sensing regions. Each region is comprised of superimposed nano-square arrays of gold $(\mathrm{Au})$ and aluminum $(\mathrm{Al})$, forming a bimetallic sensor. Nano-square shapes were chosen because (1) their design allows for easy, repeatable fabrication with electron-beam lithography, (2) the symmetrical shape is not subject to a polarisation-dependent response, and (3) the shape maximizes the surface area of coverage for a given periodicity (and thus maximize the plasmonic signal).

\subsection{Characterization of Device}

Both $\mathrm{Au}$ and $\mathrm{Al}$ can support selective functionalization of their surfaces by thiol chemistry and by silane chemistry, respectively. ${ }^{23-25,28}$ The use of organic ligands in this way has previously been reported to change the sensitivity of plasmonic sensing regions, in electronic nose applications, to certain organic molecules present in solution. ${ }^{17}$ However, only one organic ligand per sensing region was reported. In the device presented herein, the selective functionalization of each metal in the bimetallic array results in two different ligand groups on the surface. This creates a unique surface chemistry unlike that of their monometallic counter-parts and provides two distinct moieties, and thus two signals, in the same sensor area.

To test this hypothesis, one sensor was kept as native $\mathrm{Al} / \mathrm{Au}$ (Figure 1a) and the other two regions were modified by hexamethyldisilazane (HMDS) and 1-decanethiol (DT) to give an Al-HMDS / Au-DT region (Figure 1b) and by 2[methoxy (polyethyleneoxy)6-9 propyl] trimethoxysilane (PEG69) and 1H,1H,2H,2H-perfluoro-1-decanethiol (PFDT) to give an Al-PEG69 / Au-PFDT region (Figure 1c). Figure 3 shows the response in water of these three nanoarrays. The transmission peaks corresponding to $\mathrm{Al}$ are around $500 \mathrm{~nm}$ and the peaks corresponding to $\mathrm{Au}$ are around $650-700 \mathrm{~nm}$. The surface chemistry modifications alter the environment surrounding the nanostructures, resulting in a shifted 
transmission. The inset of Figure 3 shows an SEM image of one of the arrays, demonstrating the good alignment achieved between the superimposed structures. The two metals can be differentiated by the shade of grey due to their distinct electron diffraction properties, Au being 'brighter' and Al being 'darker.' 30

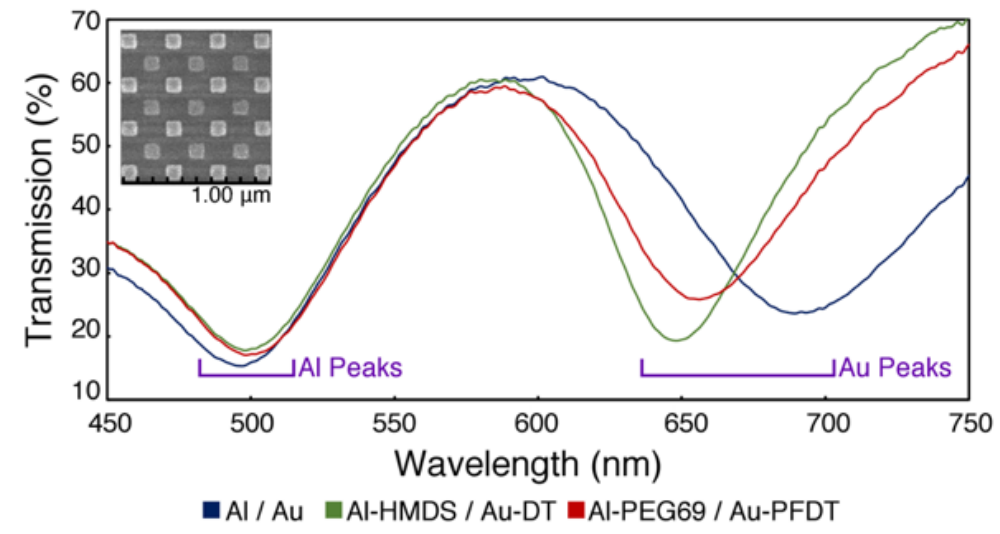

Figure 3: Characterization of bimetallic optical tongue. Transmission response of the three regions of the bimetallic optical tongue in water: Al / Au (blue), Al-HMDS / Au-DT (green), and Al-PEG69 / Au-PFDT (red). The different surface chemistry modifications in each region result in a different transmission response to the same solvent. (inset) SEM image of the native $\mathrm{Al} / \mathrm{Au}$ sensing region. The two metals can be differentiated by the shade of grey due to their distinct electron diffraction properties, Au being 'brighter' and Al being 'darker.' 30

\subsection{Organic Solvent Differentiation}

The resulting three sensing regions were then tested against varying refractive index media adjusted with acetone, methanol $(\mathrm{MeOH})$, ethanol (EtOH), and isopropyl alcohol (IPA). To determine whether the surface chemistry modifications actually alter the sensing capabilities and change the affinity for organic solvents, the resulting three pairs of transmission peaks (one from each metal component of each of the three sensing regions) were analyzed using a standard principal component analysis (PCA - a non-biased, multivariant analysis technique that can be used to delineate classes). ${ }^{22,}{ }^{29}$ For the PCA, each solvent (and subsequent repetitions of that solvent) was used as a row for the analysis and the transmission peaks corresponding to Al, Au, Al-HMDS, Au-DT, Al-PEG69, and Au-PFDT were used as the columns of that row.

a

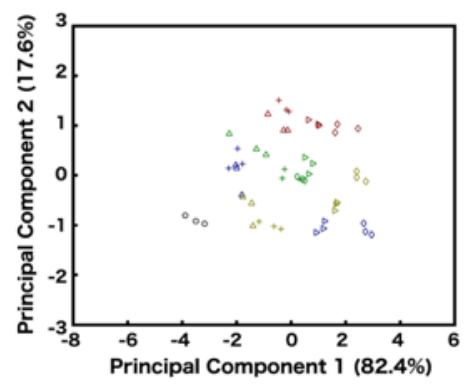

owater b

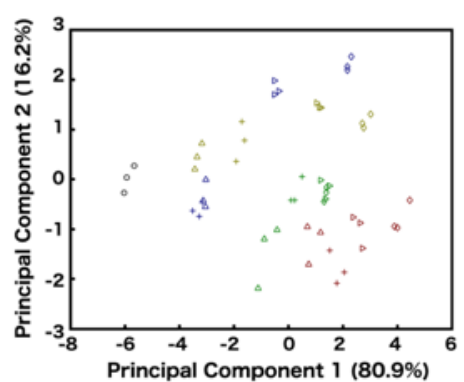

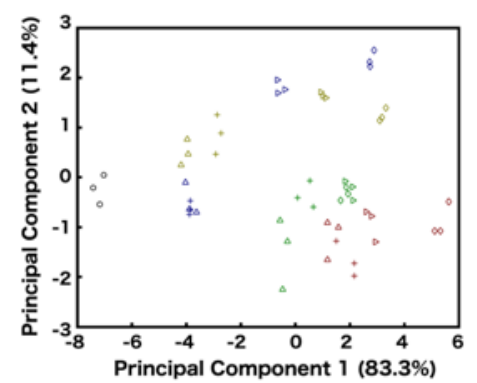

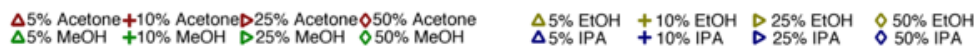

Figure 4: Organic solvent differentiation by PCA. Plot of the first and second principal components of bimetallic arrays of $\mathrm{Au} / \mathrm{Al}$ using the transmission peaks of (a) only native $\mathrm{Al} / \mathrm{Au}$, (b) $\mathrm{Al} / \mathrm{Au}$ and $\mathrm{Al}-\mathrm{HMDS} / \mathrm{Au}-\mathrm{DT}$, and (c) $\mathrm{Al} / \mathrm{Au}, \mathrm{Al}-\mathrm{HMDS} / \mathrm{Au}-\mathrm{DT}$, and $\mathrm{Al}-$ PEG69/Au-PFDT. As more elements are added to the PCA, the optical tongue is better able to distinguish the difference between water and $5 \%, 10 \%, 25 \%$ and $50 \%(\mathrm{v} / \mathrm{v})$ acetone, methanol, ethanol, and IPA.

Figure 4 shows a PCA of the first two principal components using (a) only one region ( $\mathrm{Al} / \mathrm{Au}$ ), (b) two regions ( $\mathrm{Al} / \mathrm{Au}$ and Al-HMDS / Au-DT), and (c) all three regions (Al / Au, Al-HMDS / Au-DT, Al-PEG69 / Au-PFDT) of the optical 
tongue. In each PCA, the color signifies the solvent (where black shapes represent water, red represent acetone, green represent $\mathrm{MeOH}$, yellow represent $\mathrm{EtOH}$, and blue represent IPA) and the shape signifies the percent $(\mathrm{v} / \mathrm{v})$ of solvent in water (upward triangle as $5 \%$, cross as $10 \%$, right-facing triangle as $25 \%$, and diamond as $50 \%$ ). As more elements to the sensor array are added to the PCA, further differentiation between classes emerge, which is indicated by the further separation of groupings. While there is some overlap between classification of the different concentrations within the particular of the solvents tested (i.e. $25 \%$ and $50 \% \mathrm{MeOH}, 5 \%$ and $10 \%$ IPA, etc.) there is a distinct separation between acetone, $\mathrm{MeOH}, \mathrm{EtOH}$, and IPA. To develop a high quality nanoplasmonic tongue, better inter-class separation between the different datasets of the training solutions is needed. It is hypothesised that further separation would require more partially selective sensors, since most electronic nose devices reported consist of up to 32 sensors. ${ }^{21}$ This is more than 3 times the sensor count presented, here. Regardless, the PCA is capable of clustering the different solutions by combining the response from three sensing regions, which constitutes the very basic requirement for the development of an artificial tongue.

\section{CONCLUSIONS}

In summary, we present a bimetallic nanoplasmonic approach to optical tongue/nose devices. Compared to other devices which produce one partially selective-signal per element, each element of our device provides two partially-selective signals. This effectively halves the number of elements necessary to provide the same amount of data as previous devices. We demonstrate that the partial-selectivity to organic solvents can be tuned by altering the surface chemistry of the nanostructures. We believe this technology has applications in a number of fields that rely on assays for determining multiple analytes, such as point of care diagnostics, food and drink processing, environmental monitoring, and defence.

\section{ACKNOWLEDGEMENTS}

This work was supported by The Leverhulme Trust (grant RPG-2014-343), EPSRC (grant EP/P51133X/1) and BBSRC (grant BB/N016734/1). The authors also wish to thank all the staff working in the James Watt Nanofabrication Centre for their support.

\section{REFERENCES}

(1) Jennings, W.; Shibamoto, T. Qualitative Analysis of Flavor and Fragrance Volatiles by Glass Capillary Gas Chromatography, 1 ed.; Elsevier: 2012.

(2) Ng, S. C.; Ong, T. T.; Fu, P.; Ching, C. B. Enantiomer Separation of Flavour and Fragrance Compounds by Liquid Chromatography Using Novel Urea-Covalent Bonded Methylated Beta-Cyclodextrins on Silica. Journal of Chromatography A 2002, 968 (1-2), 31-40, DOI: 10.1016/s0021-9673(02)00840-3.

(3) Welch, C. J.; Wu, N.; Biba, M.; Hartman, R.; Brkovic, T.; Gong, X.; Helmy, R.; Schafer, W.; Cuff, J.; Pirzada, Z.; Zhou, L. Greening Analytical Chromatography. Trac-Trends in Analytical Chemistry 2010, 29 (7), 667-680, DOI: 10.1016/j.trac.2010.03.008.

(4) Rodriguez-Mendez, M. L.; De Saja, J. A.; Gonzalez-Anton, R.; Garcia-Hernandez, C.; Medina-Plaza, C.; GarciaCabezon, C.; Martin-Pedrosa, F. Electronic Noses and Tongues in Wine Industry. Frontiers in Bioengineering and Biotechnology 2016, 4, DOI: 10.3389/fbioe.2016.00081.

(5) Kermani, B. G.; Schiffman, S. S.; Nagle, H. T. Performance of the Levenberg-Marquardt Neural Network Training Method in Electronic Nose Applications. Sens. Actuators, $B$ 2005, 110 (1), 13-22, DOI: 10.1016/j.snb.2005.01.008.

(6) D'Amico, A.; Pennazza, G.; Santonico, M.; Martinelli, E.; Roscioni, C.; Galluccio, G.; Paolesse, R.; Di Natale, C. An Investigation on Electronic Nose Diagnosis of Lung Cancer. Lung Cancer 2010, 68 (2), 170-176, DOI: 10.1016/j.lungcan.2009.11.003.

(7) Han, J.; Ma, C.; Wang, B.; Bender, M.; Bojanowski, M.; Hergert, M.; Seehafer, K.; Herrmann, A.; Bunz, U. H. F. A Hypothesis-Free Sensor Array Discriminates Whiskies for Brand, Age, and Taste. Chem 2017, 2 (6), 817-824, DOI: 10.1016/j.chempr.2017.04.008.

(8) Hou, C.; Dong, J.; Zhang, G.; Lei, Y.; Yang, M.; Zhang, Y.; Liu, Z.; Zhang, S.; Huo, D. Colorimetric Artificial Tongue for Protein Identification. Biosens. Bioelectron. 2011, 26 (10), 3981-3986, DOI: 10.1016/j.bios.2010.11.025. 
(9) Mimendia, A.; Gutierrez, J. M.; Leija, L.; Hernandez, P. R.; Favari, L.; Munoz, R.; del Valle, M. A Review of the Use of the Potentiometric Electronic Tongue in the Monitoring of Environmental Systems. Environmental Modelling \& Software 2010, 25 (9), 1023-1030, DOI: 10.1016/j.envsoft.2009.12.003.

(10) Persaud, K.; Dodd, G. Analysis of Discrimination Mechanisms in the Mammalian Olfactory System Using a Model Nose. Nature 1982, 299 (5881), 352-355, DOI: 10.1038/299352a0.

(11) El Barbri, N.; Llobet, E.; El Bari, N.; Correig, X.; Bouchikhi, B. Electronic Nose Based on Metal Oxide Semiconductor Sensors as an Alternative Technique for the Spoilage Classification of Red Meat. Sensors 2008, 8 (1), 142-156, DOI: 10.3390/s8010142.

(12) Turner, A. P. F.; Magan, N. Electronic Noses and Disease Diagnostics. Nature Reviews Microbiology 2004, 2 (2), 161-166, DOI: $10.1038 /$ nrmicro823.

(13) Brenner, H. Long-Term Survival Rates of Cancer Patients Achieved by the End of the 20th Century: A Period Analysis. Lancet 2002, 360 (9340), 1131-1135, DOI: 10.1016/s0140-6736(02)11199-8.

(14) Vosshall, L. B.; Stocker, R. E. Molecular Architecture of Smell and Taste in Drosophila. Annual Review of Neuroscience 2007, 30, 505-533, DOI: 10.1146/annurev.neuro.30.051606.094306.

(15) Vlasov, Y.; Legin, A.; Rudnitskaya, A.; Di Natale, C.; D'Amico, A. Nonspecific Sensor Arrays ("Electronic Tongue") for Chemical Analysis of Liquids (Iupac Technical Report). Pure and Applied Chemistry 2005, 77 (11), 19651983, DOI: 10.1351/pac200577111965.

(16) Goodner, K. L.; Dreher, J. G.; Rouseff, R. L. The Dangers of Creating False Classifications Due to Noise in Electronic Nose and Similar Multivariate Analyses. Sens. Actuators, B 2001, 80 (3), 261-266, DOI: 10.1016/s09254005(01)00917-0.

(17) Peng, G.; Tisch, U.; Adams, O.; Hakim, M.; Shehada, N.; Broza, Y. Y.; Billan, S.; Abdah-Bortnyak, R.; Kuten, A.; Haick, H. Diagnosing Lung Cancer in Exhaled Breath Using Gold Nanoparticles. Nature Nanotechnology 2009, 4 (10), 669-673, DOI: 10.1038/nnano.2009.235.

(18) Maier, S. A. Plasmonics: Fundamentals and Applications, Springer Science+Business Media LLC: New York, 2007.

(19) Peng, G.; Hakim, M.; Broza, Y. Y.; Billan, S.; Abdah-Bortnyak, R.; Kuten, A.; Tisch, U.; Haick, H. Detection of Lung, Breast, Colorectal, and Prostate Cancers from Exhaled Breath Using a Single Array of Nanosensors. British Journal of Cancer 2010, 103 (4), 542-551, DOI: 10.1038/sj.bjc.6605810.

(20) Li, W.; Liu, H.; Xie, D.; He, Z.; Pi, X. Lung Cancer Screening Based on Type-Different Sensor Arrays. Sci. Rep. 2017, 7, DOI: 10.1038/s41598-017-02154-9.

(21) Cellini, A.; Blasioli, S.; Biondi, E.; Bertaccini, A.; Braschi, I.; Spinelli, F. Potential Applications and Limitations of Electronic Nose Devices for Plant Disease Diagnosis. Sensors 2017, 17 (11), DOI: 10.3390/s17112596.

(22) Askim, J. R.; Mahmoudi, M.; Suslick, K. S. Optical Sensor Arrays for Chemical Sensing: The Optoelectronic Nose. Chem. Soc. Rev. 2013, 42 (22), 8649-8682, DOI: 10.1039/c3cs60179j.

(23) Srisombat, L.; Jamison, A. C.; Lee, T. R. Stability: A Key Issue for Self-Assembled Monolayers on Gold as ThinFilm Coatings and Nanoparticle Protectants. Colloids and Surfaces a-Physicochemical and Engineering Aspects 2011, 390 (1-3), 1-19, DOI: 10.1016/j.colsurfa.2011.09.020.

(24) Nicosia, C.; Huskens, J. Reactive Self-Assembled Monolayers: From Surface Functionalization to Gradient Formation. Materials Horizons 2014, 1 (1), 32-45, DOI: 10.1039/c3mh00046j.

(25) Jani, A. M. M.; Anglin, E. J.; McInnes, S. J. P.; Losic, D.; Shapter, J. G.; Voelcker, N. H. Nanoporous Anodic Aluminium Oxide Membranes with Layered Surface Chemistry. Chem. Commun. (Cambridge) 2009, (21), 30623064, DOI: 10.1039/b901745c.

(26) Rumble, J. R.; Lide, D. R.; Bruno, T. J. Crc Handbook of Chemistry \& Physics, 98 ed.; CRC Press: Boca Raton, USA, 2017.

(27) Palik, E. D. Handbook of Optical Constants of Solids, Academic Press: 1997; Vol. I-III.

(28) Sperling, J. R.; Macias, G.; Neale, S. L.; Clark, A. W. Multilayered Nanoplasmonic Arrays for Self-Referenced Biosensing. ACS Applied Materials \& Interfaces 2018, DOI: 10.1021/acsami.8b12604.

(29) Wold, S.; Esbensen, K.; Geladi, P. Principal Component Analysis. Chemometrics and Intelligent Laboratory Systems 1987, 2 (1-3), 37-52, DOI: 10.1016/0169-7439(87)80084-9.

(30) Goldstein, J. I.; Newbury, D. E.; Micheal, J. R.; Ritchie, N. W. M.; Scott, J. H. J.; Joy, D. C. Scanning Electron Microscopy and X-Ray Microanalysis, 4 ed.; Springer: 2017. 